\title{
Geometric structures of Al nanoparticles adsorbed on graphene under an external electric field
}

\author{
Manaho Matsubara* and Susumu Okada ${ }^{\dagger}$ \\ Graduate School of Pure and Applied Sciences, University of Tsukuba, Tsukuba, Ibaraki 305-8571, Japan
}

\begin{abstract}
Using the density functional theory combined with the effective screening medium method, we studied geometric structures of $\mathrm{Al}$ nanoparticles adsorbed on graphene surfaces under electron/hole injection by a counter electrode. The equilibrium spacing between graphene and an Al nanoparticle is sensitive to their mutual arrangement with respect to the electrode, carrier concentration, and carrier species. For most cases, owing to the Coulomb attractive interaction between accumulated carriers in graphene or an $\mathrm{Al}$ nanoparticle and the counter electrode, the Al nanoparticle is desorbed from the graphene surfaces at the carrier concentration of approximately 0.5 e or $0.5 \mathrm{~h}$ per unit cell.
\end{abstract}

\section{Introduction}

Graphene has been receiving much attention in the field of not only low-dimensional science but also electronic device engineering owing to its unique structural and electronic properties. ${ }^{1,2)}$ Pairs of linear dispersion bands at six corners of the Brillouin zone cause the unusual Hall effect ${ }^{3,4)}$ and a remarkable carrier mobility ${ }^{5-7)}$ of up to 200,000 $\mathrm{cm}^{2} \mathrm{~V}^{-1} \mathrm{~s}^{-1}$, which make graphene an emerging material for high-speed-switching electronic devices. $\left.{ }^{8}\right)$ In addition to its intrinsic unique electronic properties, graphene exhibits further variation in its electronic structure by forming hybrid structures with foreign materials and the external environment. ${ }^{9-13)}$ Graphene no longer possesses linear dispersion bands when it is adsorbed on insulating substrates, ${ }^{14-17)}$ metal surfaces, ${ }^{18}$ ) and defective graphene layers, ${ }^{19)}$ owing to the modulation of the electrostatic potential on graphene by foreign materials. An external electric field also modulates the electronic structure of graphene and its hybrids. ${ }^{20-23)}$ In electronic devices, the hybrid structures of graphene with foreign materials, such as environmental molecules and nanoparticles, and an external electric field are essential for controlling their functionalities. In our previous work, such hybrids under an electric field endow versatile electronic properties

\footnotetext{
${ }^{*}$ E-mail: mmatsubara@comas.frsc.tsukuba.ac.jp

†E-mail: sokada@comas.frsc.tsukuba.ac.jp
} 
that are absent in an isolated graphene. ${ }^{24,25)}$ In graphene thin films, one of whose layers possesses defects, the Fermi level is sensitive to the relative arrangement of the defective graphene layer and the carrier species, leading to the Fermi level pinning for hole doping. ${ }^{24)}$ Charged nanoparticles adsorbed on graphene surfaces also provide unique electronic properties under carrier injection by the gate electrode. ${ }^{25)}$

Despite the fact that the electronic structure of such hybrids under an electric field has been elucidated, the detailed energetics and geometric structures of hybrids under the electric field have not been well examined in detail yet. Thus, in this work, we aim to elucidate the geometric structures of $\mathrm{Al}$ nanoparticles adsorbed on graphene under excess electron or hole injection by a counter electrode using the density functional theory (DFT) combined with the effective screening medium (ESM) method. Our calculations showed that the equilibrium position of $\mathrm{Al}$ nanoparticles under excess electron/hole injection is sensitive to the carrier species and the arrangement of $\mathrm{Al}$ nanoparticles with respect to the counter electrode. The electron injection in the hybrids increases the equilibrium spacing between the nanoparticle and graphene, irrespective of the arrangement of the nanoparticles relative to the electrode. In contrast, for hole doping, the equilibrium spacing monotonically decreases with increasing number of holes when the nanoparticles are adsorbed on the surface of graphene opposite to the electrode, while it has a minimum at a hole concentration that depends on the nanoparticle size when the nanoparticles are sandwiched between graphene and the electrode.

\section{Calculation method and structural models}

All calculations were performed on the basis of DFT ${ }^{26,27)}$ using the STATE package. ${ }^{28)}$ To express the exchange correlation potential among the interacting electrons, local density approximation (LDA) was applied with the Perdew-Wang functional form ${ }^{29}$ ) fitting to the quantum Monte Carlo results on a homogeneous electron gas, ${ }^{30)}$ since the LDA is known to qualitatively express weak interactions in carbon nanomaterials. ${ }^{31}$ ) Note that the LDA usually overbinds the materials interacting via noncovalent interactions. We used ultrasoft pseudopotentials generated using the Vanderbilt scheme to describe the interaction between nuclei and electrons. ${ }^{32)}$ The valence wave functions and charge density were expanded in terms of the plane-wave basis set with cutoff energies of 25 and 225 Ry, respectively. Brillouin zone integration was performed with the $\Gamma$-centered $8 \times 8 \times 1$ uniform $\boldsymbol{k}$-mesh for self-consistent electronic structure calculations for graphene with $4 \times 4$ lateral periodicity, which corresponds to the $32 \times 32 \times 1 \boldsymbol{k}$-mesh 
(a)

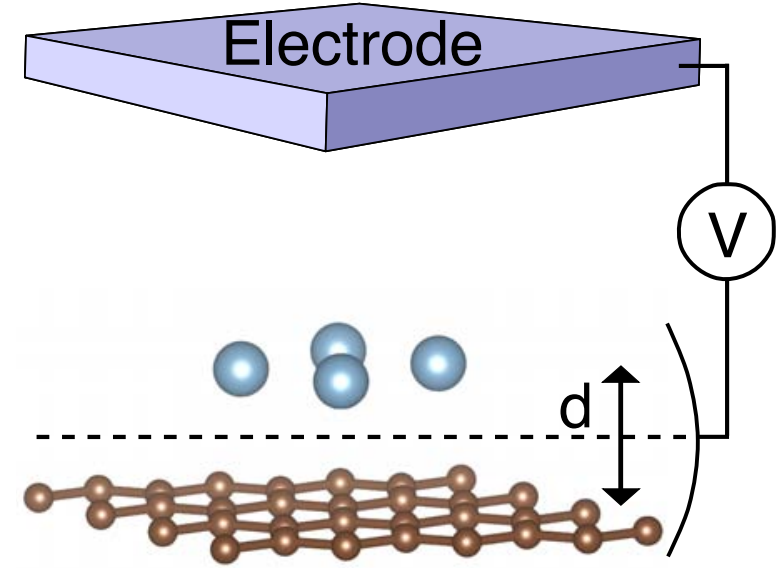

(b)

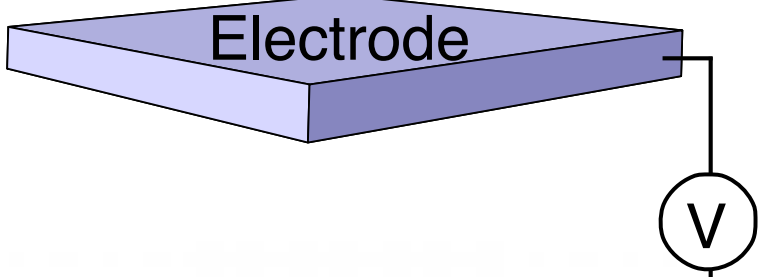

Fig. 1. (Color online) Structural models of Al nanoparticles adsorbed on graphene with the FET structure with the (a) electrode-Al-graphene and (b) electrode-graphene- $\mathrm{Al}$ arrangements. Large and small balls denote $\mathrm{Al}$ and $\mathrm{C}$ atoms, respectively. Slabs situated above the hybrid consisting of graphene and $\mathrm{Al}$ nanoparticles denote the counter electrodes, which are simulated by the ESM.

in a primitive $1 \times 1$ cell of graphene, resulting in sufficient convergence for the geometric and electronic structures. According to the choice of the $\boldsymbol{k}$-mesh, the Brillouin zone integration contains the $\mathrm{K}$ and $\mathrm{K}$ ' points, allowing us to discuss the phenomena associated with the Dirac point. All atoms were fully optimized until the remaining force acting on each atom was less than $0.005 \mathrm{Ry} / \AA$ under a fixed lateral lattice constant of $9.83 \AA$ corresponding to the experimental value of a $4 \times 4$ lateral cell of graphene.

In this work, we consider a graphene field-effect transistor (FET) in which $\mathrm{Al}$ nanoparticles are adsorbed on the graphene surfaces with two different adsorption arrangements with respect to the electrode (Fig. 1), because the $\mathrm{Al}$ nanoparticles are possible and representative charged impurities in a FET structure generated by the $\mathrm{Al}$ electrode. We consider square $\mathrm{Al}_{4}$ and triangular $\mathrm{Al}_{3}$ nanoparticles as representative impurity nanoparticles in the graphene FET, which are adsorbed on graphene per $4 \times 4$ lateral periodicity. To investigate the geometric and electronic structures of graphene with metal nanoparticles under electron and hole injection, we adopted the ESM method to solve the Poisson equation under a boundary condition in which a planar metal electrode and a half-infinite vacuum are situated at two-cell boundaries parallel to the graphene layer (Fig. 1). ${ }^{33)}$ Electrons and holes were injected by the planar counter gate electrode situated at the cell boundary dislodged by $6.35 \AA$ from the center of graphene 
(a)

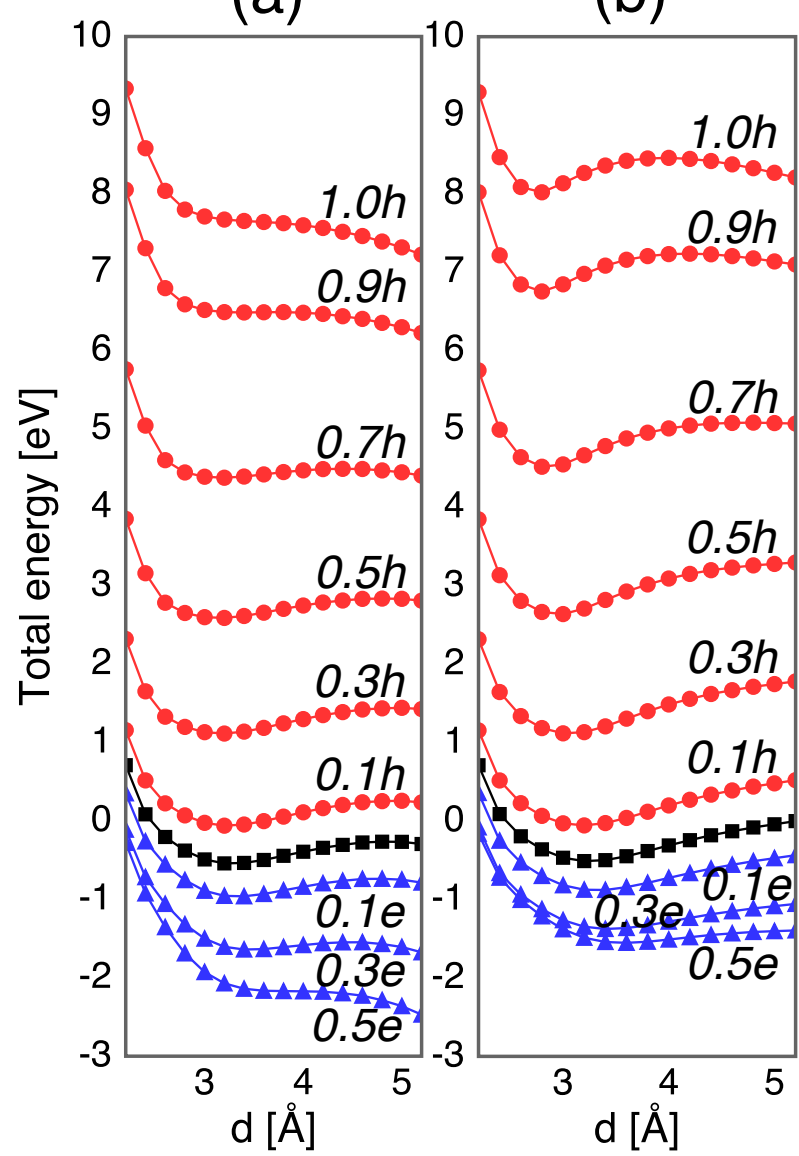

(c)

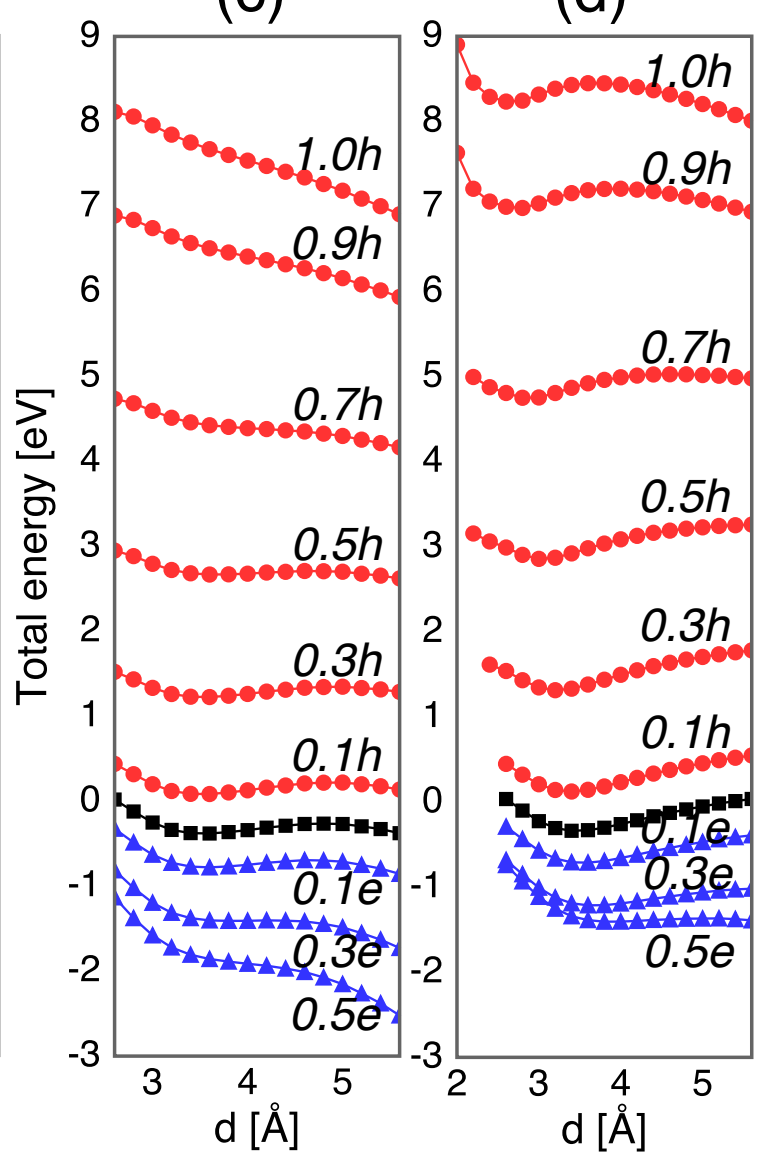

Fig. 2. (Color online) Total energies of $\mathrm{Al}_{4}$ adsorbed on graphene with (a) electrode-Al-graphene and (b) electrode-graphene-Al arrangements as a function of spacing between $\mathrm{Al}_{4}$ and graphene at carrier concentrations from 0.5 e to $1.0 \mathrm{~h}$. Total energies of $\mathrm{Al}_{3}$ adsorbed on graphene with (c) electrode-Al-graphene and (d) electrode-graphene-Al arrangements as a function of the spacing between $\mathrm{Al}_{3}$ and graphene at carrier concentrations from 0.5 e to $1.0 \mathrm{~h}$. In each panel, filled squares, circles, and triangles denote the total energies under neutral, hole-doped, and electron-doped conditions, respectively.

and $\mathrm{Al}$ nanoparticles. The electrode was simulated by the ESM with an infinite relative permittivity.

\section{Results and discussion}

Figure 2 shows the total energy of Al nanoparticles adsorbed on graphene at each carrier concentration as a function of the spacing between them. The total energy, $E$, is calculated as

$$
E=E_{\mathrm{Gra} / \mathrm{Al}}^{\boldsymbol{F}}-E_{\mathrm{Gra}}^{0}-E_{\mathrm{Al}}^{0},
$$


where $E_{\mathrm{Gra} / \mathrm{Al}}^{\boldsymbol{F}}, E_{\mathrm{Gra}}^{0}$, and $E_{\mathrm{Al}}^{0}$ are the total energies of graphene adsorbing Al nanoparticles under the electric field, an isolated graphene without the external field, and an isolated Al nanoparticle without the field, respectively. The energy minima under the electron/hole doping are shallower than those under the neutral condition except for hole doping in structures in which the Al nanoparticles are adsorbed on the surface of graphene opposite to the electrode. This observation indicates that the carrier injection in the hybrids comprising graphene and $\mathrm{Al}$ nanoparticles basically decreases the binding energy of the $\mathrm{Al}$ nanoparticles to graphene.

For electron doping, the energy minimum vanishes at an electron concentration of $0.5 \mathrm{e} /$ cell, indicating that the electron injection desorbs the $\mathrm{Al}$ nanoparticles from graphene. The disappearance of the energy minimum under electron doping is ascribed to the Coulomb interaction arising from the additional electrons injected by the counter electrode. An attractive Coulomb interaction between negatively charged Al nanoparticles or graphene and the positively charged counter electrode overcomes the binding interaction between $\mathrm{Al}$ nanoparticles and graphene arising from the quantum mechanical effect.

For hole doping, the energy profile is sensitive to the arrangement of the Al nanoparticle relative to the electrode. When the Al nanoparticles are situated on the electrode side, the injected holes induce a strong attractive Coulomb interaction between the nanoparticles and the negatively charged electrode, resulting in an increase in the spacing between graphene and the Al nanoparticles. On the other hand, the equilibrium spacing monotonically decreases with increasing hole concentration when the $\mathrm{Al}$ nanoparticle is located on the surface of graphene opposite to the electrode. Furthermore, the depth of the energy minimum is approximately the same as that under the neutral condition.

Figure 3 shows the equilibrium spacing between graphene and the $\mathrm{Al}$ nanoparticles as a function of carrier concentration. As stated above, the equilibrium spacing between the $\mathrm{Al}$ nanoparticles and graphene monotonically increases with increasing number of electrons, irrespective of the $\mathrm{Al}$ nanoparticle arrangement and size. Furthermore, the $\mathrm{Al}$ nanoparticles are desorbed from graphene surfaces at approximately 0.5 e doping with the electrode-Al-graphene arrangement, owing to the Coulomb attractive interaction between Al nanoparticles and the counter electrode. For the hole doping, the equilibrium spacing depends on the relative arrangements of the $\mathrm{Al}$ nanoparticles with respect to the counter gate electrode. The equilibrium spacing monotonically decreases with 
(a)

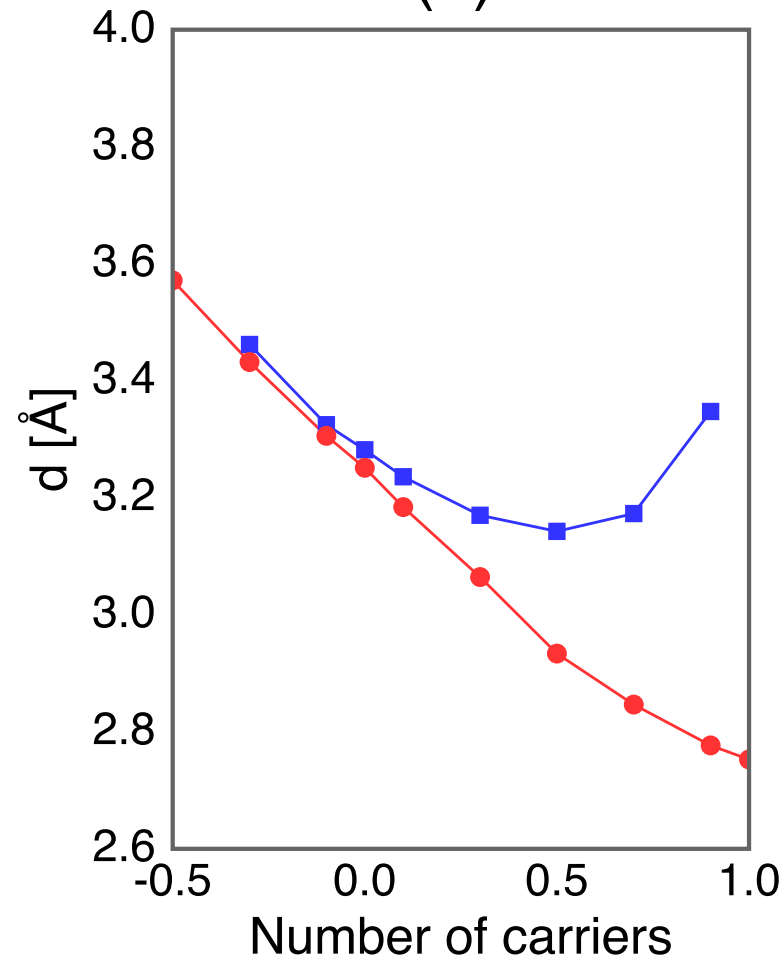

(b)

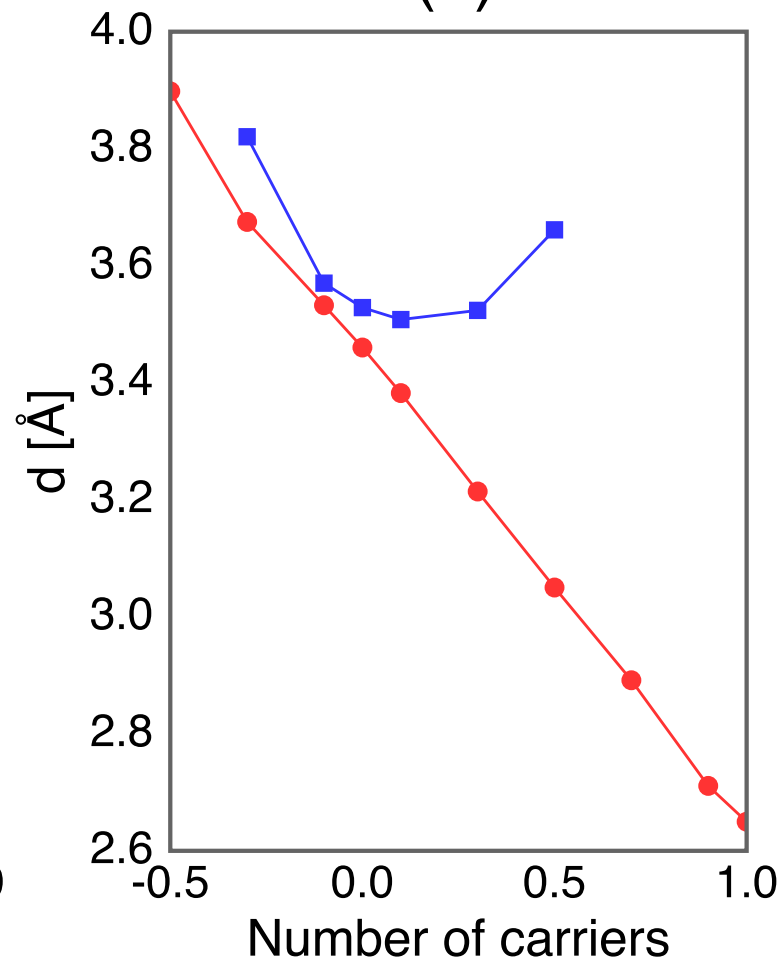

Fig. 3. (Color online) Equilibrium spacing between Al nanoparticles and graphene as a function of carrier concentration for (a) $\mathrm{Al}_{4}$ and (b) $\mathrm{Al}_{3}$ nanoparticles. In each panel, the circles and squares denote the spacing for the electrode-graphene-Al and electrode-Al-graphene arrangements, repsectively.

increasing the number of holes when the $\mathrm{Al}$ nanoparticles are located at the surface of graphene opposite to the electrode. The equilibrium spacing saturates at approximately 2.6 and $2.4 \AA$ for $\mathrm{Al}_{4}$ and $\mathrm{Al}_{3}$ nanoparticles, respectively. In the case of $\mathrm{Al}$ nanoparticles sandwiched between graphene and the electrode, the equilibrium spacing has minima at the hole concentrations of $0.5 \mathrm{~h}$ and $0.1 \mathrm{~h}$ for $\mathrm{Al}_{4}$ and $\mathrm{Al}_{3}$, respectively. Then, a further increase in hole concentration leads to the desorption of the Al nanoparticles at the hole concentrations of $0.9 \mathrm{~h}$ and $0.5 \mathrm{~h}$ for $\mathrm{Al}_{4}$ and $\mathrm{Al}_{3}$ nanoparticles, respectively, as in the case of the $\mathrm{Al}$ nanoparticles under electron doping.

Figure 4 shows the distribution of the carriers accumulated in the Al nanoparticles adsorbed on graphene under equilibrium spacing and carrier concentrations of $0.3 \mathrm{e}$ and $0.5 \mathrm{~h}$. For the electron doping, the accumulated electrons are primarily distributed on the $\mathrm{Al}$ nanoparticles when the $\mathrm{Al}$ nanoparticles are sandwiched between graphene and the electrode [Figs. 4(a) and 4(c)]. The electrons are distributed on the graphene layer when the graphene layer is situated on the electrode side. The equilibrium spac- 
(a)
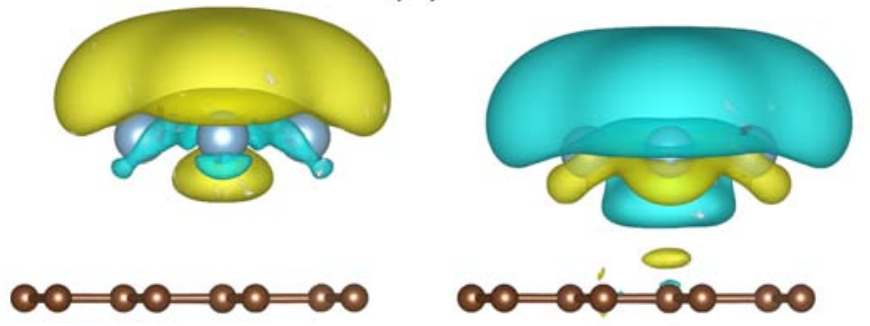

(b)
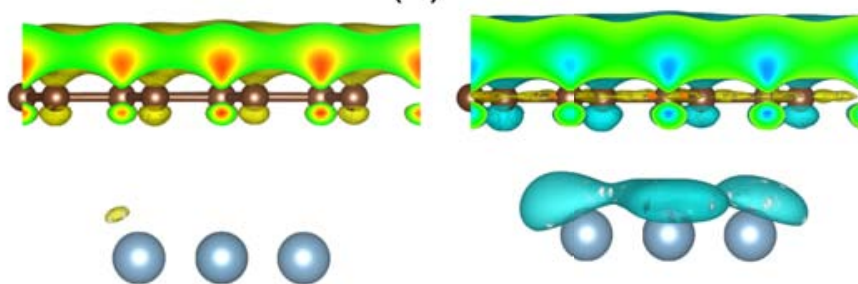

(c)
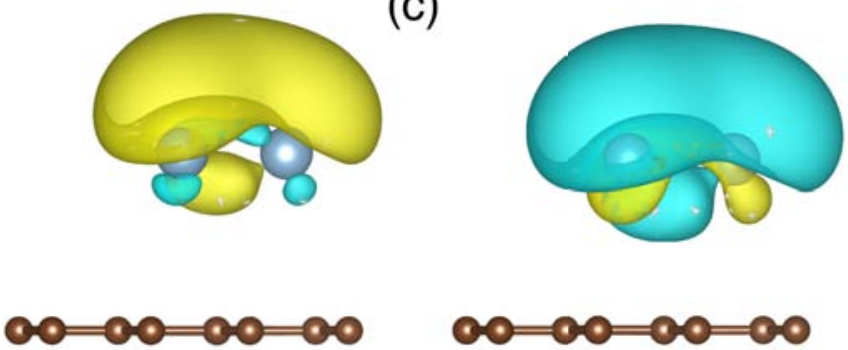

(d)

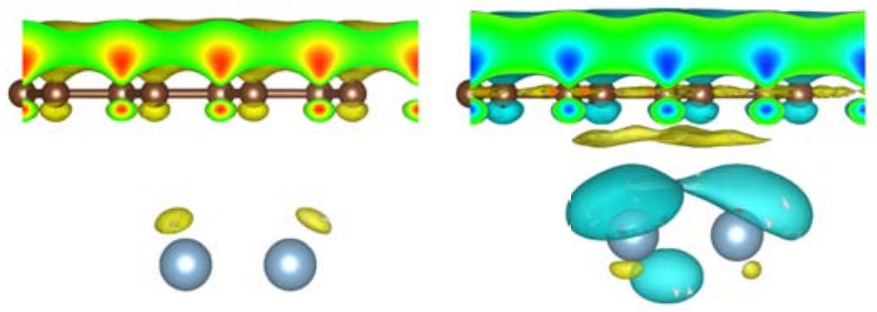

Fig. 4. (Color online) Isosurfaces of the distribution of the accumulated electrons and holes injected by the gate electrode in graphene- $\mathrm{Al}_{4}$ hybrids in which $\mathrm{Al}_{4}$ is adsorbed (a) above and (b) below the graphene layer with respect to the electrode. Isosurfaces of the distribution of the accumulated electrons and holes injected by the gate electrode in graphene- $\mathrm{Al}_{3}$ hybrids, in which $\mathrm{Al}_{3}$ is adsorbed (c) above and (d) below the graphene layer. In each figure, the left and right panels show the isosurfaces at 0.3 e and $0.5 \mathrm{~h}$ doping, respectively. Yellow and blue isosurfaces denote the region where the electrons and holes increase, respectively. Small and large balls denote $\mathrm{C}$ and $\mathrm{Al}$ atoms, respectively.

ing between graphene and the $\mathrm{Al}$ nanoparticles tends to increase under the electron doping. The localized nature of the accumulated electrons causes a strong attractive Coulomb interaction with the counter electrode, causing the increase in the equilibrium 
spacing. For the hole injection by the electrode, the equilibrium spacing depends on the $\mathrm{Al}$ nanoparticle arrangement. The $\mathrm{Al}$ nanoparticles are desorbed from graphene at high hole concentrations when the $\mathrm{Al}$ nanoparticles are sandwiched between graphene and the electrode. In this case, the accumulated holes are localized on the Al nanoparticles, causing a strong attractive interaction with the counter electrode as in the case of electron doping. In contrast, when graphene is situated on the electrode side, the accumulated holes exhibit a complex nature. The holes are distributed not only on graphene but also on Al nanoparticles [Figs. 4(b) and 4(d)]. In addition, the electrons and holes coexist in the spacing between graphene and Al nanoparticle. The substantial charge distribution between graphene and $\mathrm{Al}$ nanoparticles is caused by the orbital hybridization between graphene and $\mathrm{Al}$ nanoparticles.

Figure 5 shows the squared wave function associated with the Al nanoparticles and graphene near the Fermi level at the K point. Under equilibrium spacing, the electron states of the Al nanoparticle are substantially hybridized with those of graphene under hole doping with the electrode-graphene-Al arrangement [Figs. 5(d) and 5(h)]. The hybridization between the electron states of graphene and Al nanoparticles induces the complex carrier distribution between graphene and $\mathrm{Al}$ nanoparticles upon hole doping. Furthermore, the hybridization results in the small equilibrium spacing between the $\mathrm{Al}$ nanoparticles and the graphene layer under hole doping. The results indicate that the hole injection in graphene hybrids with nanoparticles or molecules may generate novel complexes in which adsorbed materials are tightly bound to graphene. Furthermore, the electric field may also promote the chemical reactions of the nanoparticles on graphene.

Usually, the injected carriers are primarily accommodated in the electron states of graphene or the Al nanoparticles that are situated on the electrode side. Accordingly, the electron states of $\mathrm{Al}$ nanoparticles (the $\alpha$ and $\beta$ states) shift upward and downward upon hole and electron injection and the Dirac point (the $\gamma$ state) is pinned approximately at an energy of $-4.6 \mathrm{eV}$ when the $\mathrm{Al}$ nanoparticle is sandwiched between the electrode and graphene [Figs. 6(a) and 6(c)]. In contrast, when the $\mathrm{Al}$ nanoparticles are adsorbed on the graphene opposite to the electrode, the carriers are accumulated on the graphene, which causes the downward or upward shift of the Dirac point upon electron or hole doping, respectively, while the electron states associated with the Al nanoparticles are insensitive to the electron doping [Figs. 6(b) and 6(d)]. However, for the hole doping, the electron states of Al nanoparticles simultaneously shift upward following the upward shift of the Dirac point (the $\gamma$ state). According to the upward shift of the electronic 
(a)

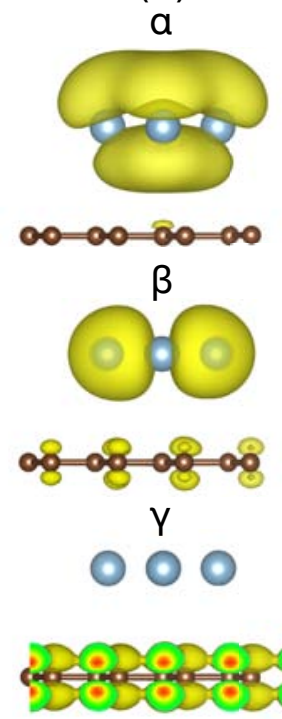

(e)

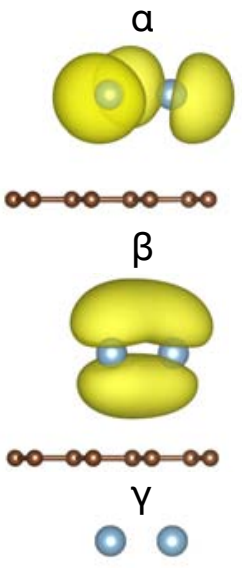

$60=060=00$ (b)

a
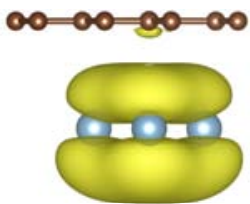

$\beta$
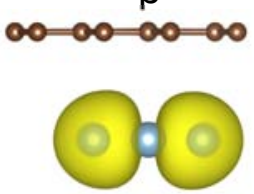

Y

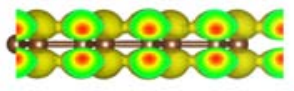

$\circ \circ \bigcirc$

(f)

a
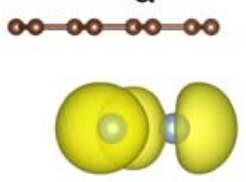

$\beta$
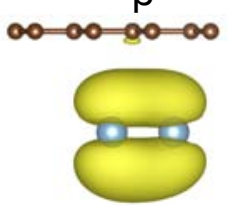

$Y$

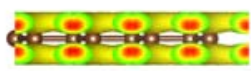

- (c)

a

(d)

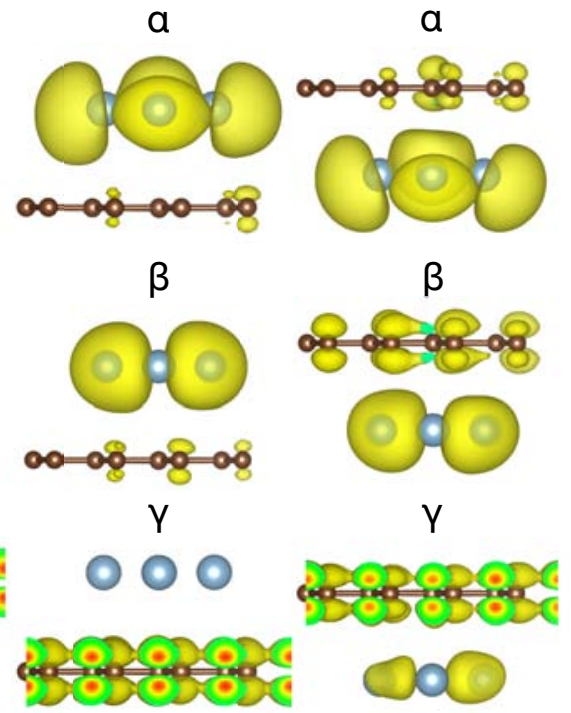

(h)

(g)
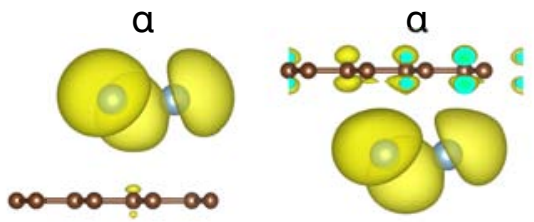

$\beta$

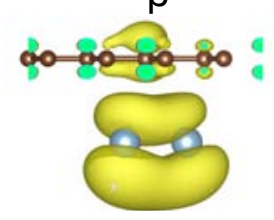

Y

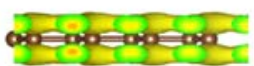

1)

Fig. 5. (Color online) Squared wave function of the electronic states near the Fermi level of graphene adsorbing $\mathrm{Al}_{4}$ with (a) electrode-Al-graphene arrangement at 0.3 e doping, (b) electrode-graphene-Al arrangement at 0.3 e doping, (c) electrode-Al-graphene at $0.5 \mathrm{~h}$ doping, (d) electrode-graphene-Al at $0.5 \mathrm{~h}$ doping. Squared wave function of the electronic states near the Fermi level of graphene adsorbing $\mathrm{Al}_{3}$ with (e) electrode-Al-graphene arrangement at 0.3 e doping, (f) electrode-graphene-Al arrangement at 0.3 e doping, (g) electrode-Al-graphene at $0.5 \mathrm{~h}$ doping, (h) electrode-graphene-Al at $0.5 \mathrm{~h}$ doping. The labels $\alpha$ and $\beta$ indicate the electron states associated with the Al nanoparticles near the Fermi level. $\gamma$ indicates the electron state of graphene at the Dirac cone.

states of the $\mathrm{Al}$ nanoparticles, the holes are injected not only in graphene but also in the Al nanoparticles. The substantial upward shift of the Al nanoparticle is ascribed to the hybridization of wave functions between the $\mathrm{Al}$ nanoparticle and graphene within 
(a)

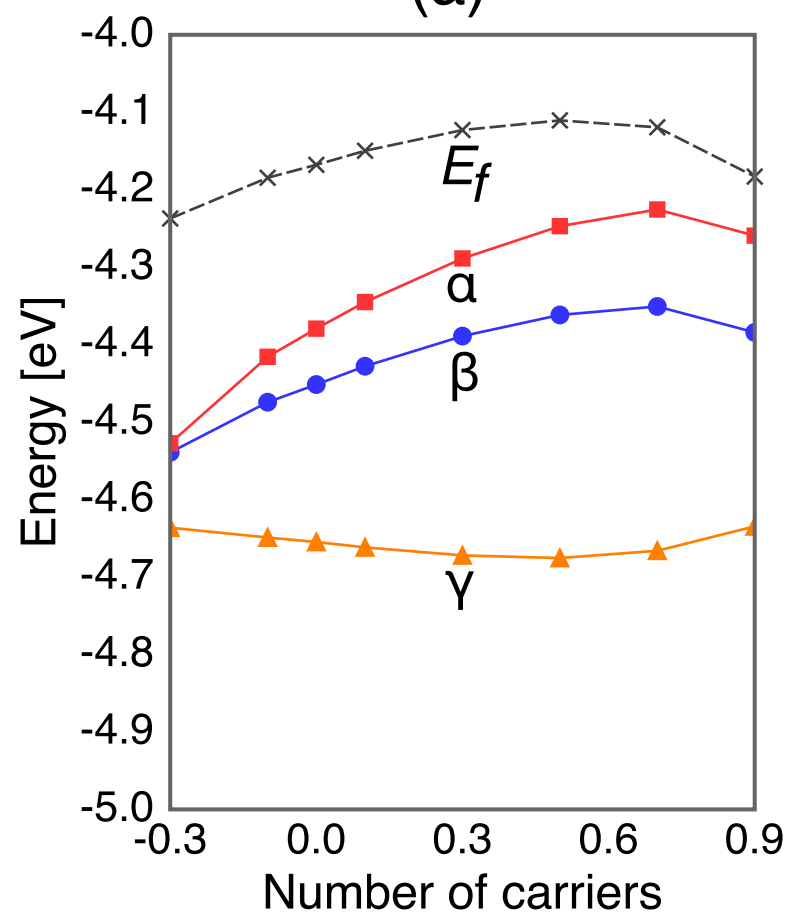

(c)

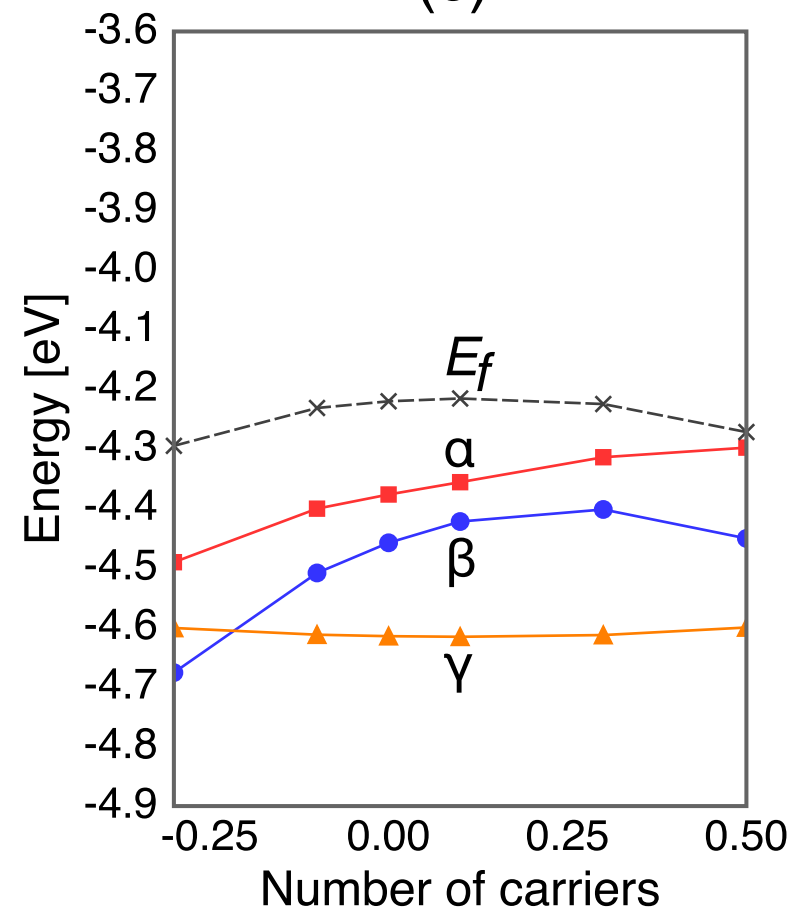

(b)

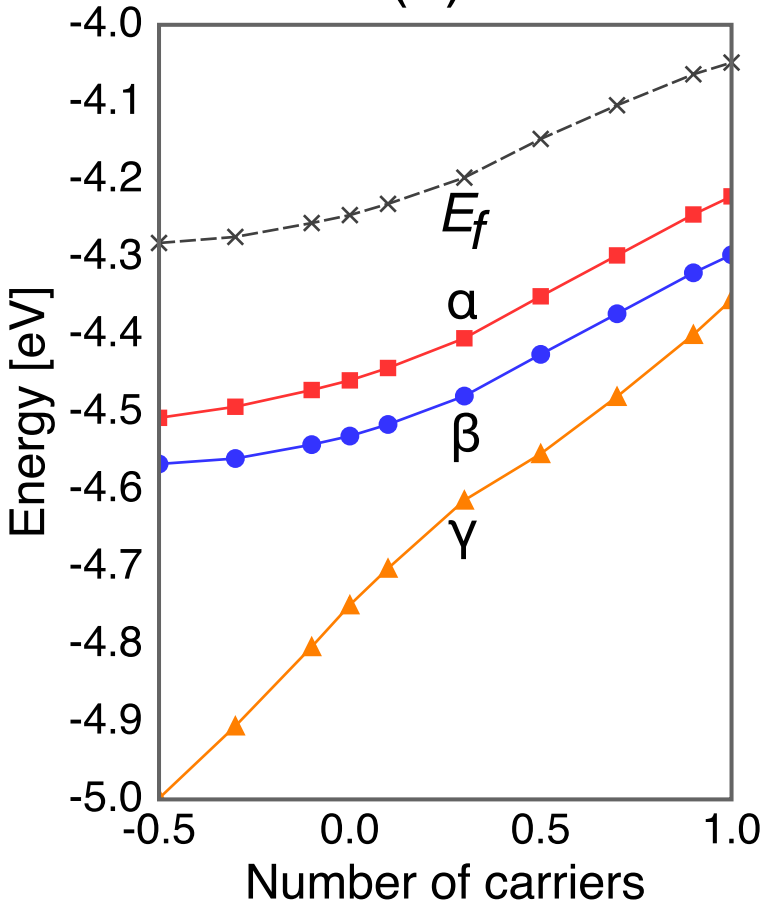

(d)

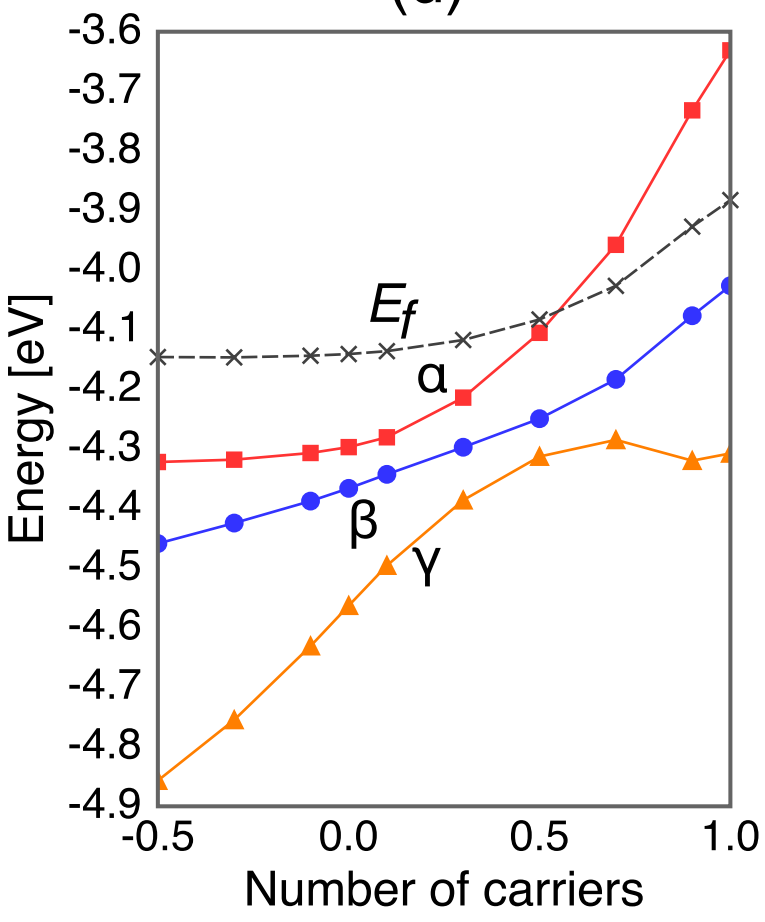

Fig. 6. (Color online) Energy values of the electronic states near the Fermi level of the $\mathrm{Al}$ nanoparticles adsorbed on graphene as a function of carrier concentration with (a) electrode- $\mathrm{Al}_{4}$-graphene, (b) electrode-graphene- $\mathrm{Al}_{4}$, (c) electrode- $\mathrm{Al}_{3}$-graphene, and (d) electrode-graphene- $\mathrm{Al}_{3}$ arrangements. The labels in each panel correspond to the electronic states shown in Fig. 5. 
the small equilibrium spacing.

\section{Summary}

We studied the geometric structures of Al nanoparticles adsorbed on graphene under excess electron or hole injection by a counter electrode using DFT combined with the ESM method. Our calculations showed that the stable equilibrium position of the $\mathrm{Al}$ nanoparticles under excess electron/hole injection is sensitive to the carrier species and the arrangement of them with respect to the counter electrode. The electron injection in hybrids increases the equilibrium spacing between the nanoparticle and graphene, irrespective of the arrangement of the nanoparticles relative to the electrode. In contrast, for hole doping, the equilibrium spacing monotonically decreases with increasing number of holes when the nanoparticles are situated on the vacuum side, while the equilibrium spacing has a minimum under the hole concentration that depends on the nanoparticle size when the nanoparticle is sandwiched between graphene and the electrode. The present results may give theoretical insights into a possible procedure to control the geometric structure of charged impurities in graphene-based FET structures.

\section{Acknowledgement}

This work was supported by JST-CREST Grant Number JPMJCR1532 from the Japan Science and Technology Agency, JSPS KAKENHI Grant Number JP17H01069, JP16H00898, and JP16H06331 from Japan Society for the Promotion of Science, and the Joint Research Program on Emission Energy Research, Institute of Advanced Energy, Kyoto University. A part of the calculations was performed on an NEC SX-Ace at the Cybermedia Center at Osaka University and on an SGI ICE XA/UV at the Institute of Solid State Physics, The University of Tokyo. 


\section{References}

1) M. S. Dresselhaus and G. Dresselhaus, Adv. Phys. 30, 139 (1981).

2) A. H. C. Neto, F. Guinea, N. M. R. Peres, K. S. Novoselov, and A. K. Geim, Rev. Mod. Phys. 81, 109 (2009).

3) K. S. Novoselov, A. K. Geim, S. V. Morozov, D. Jiang, M. I. Katsnelson, I. V. Grigorieva, S. V. Dubonos, and A. A. Firsov, Nature 438, 197 (2005).

4) Y. Zhang, Y.-W. Tan, H. L. Stormer, and P. Kim, Nature 438, 201 (2005).

5) K. I. Bolotin, K. J. Sikes, Z. Jiang, M. Klima, G. Fudenberg, J. Hone, P. Kim, and H. L. Stormer, Solid State Commun. 146, 351 (2008).

6) J. B. Oostinga, H. B. Heersche, X. Liu, A. F. Morpurgo, and L. M. K. Vandersypen, Nat. Mater. 7, 151 (2007).

7) Y. Zhang, T. Tang, C. Girit, Z. Hao, M. C. Martin, A. Zettl, M. F. Crommie, Y. R. Shen, and F. Wang, Nature 459, 820 (2009).

8) M. F. Craciun, S. Russo, M. Yamamoto, J. B. Oostinga, A. F. Morpurgo, and S. Tarucha, Nat. Nanotechnol. 4, 383 (2009).

9) N. Igami, K. Nakada, and S. Okada, Synth. Met. 121, 1233 (2001).

10) R. Balog, B. Jorgensen, L. Nilsson, M. Andersen, E. Rienks, M. Bianchi, M. Fanetti, E. Laegsgaard, A. Baraldi, S. Lizzit, Z. Sljivancanin, F. Besenbacher, B. Hammer, T. G. Pedersen, P. Hofmann, and L. Hornekaer, Nat. Mater. 9, 315 (2010).

11) J. O. Sofo, A. S. Chaudhari, and G. D. Barber, Phys. Rev. B 75, 153401 (2007).

12) J. Zhou, M. M. Wu, X. Zhou, and Q. Sun, Appl. Phys. Lett. 95, 103108 (2009).

13) N. T. Cuong, M. Otani, and S. Okada, Appl. Phys. Lett. 101, 233106 (2012).

14) S. Y. Zhou, G.-H. Gweon, A. V. Fedorov, P. N. First, W. A. de Heer, D.-H. Lee, F. Guinea, A. H. C. Neto, and A. Lanzara, Nat. Mater. 6, 770 (2007).

15) A. Mattausch and O. Pankratov, Phys. Rev. Lett. 99, 076802 (2007).

16) N. T. Cuong, M. Otani, and S. Okada, Phys. Rev. Lett. 106, 106801 (2011).

17) K. Kamiya, N. Umezawa, and S. Okada, Phys. Rev. B 83, 153413 (2011).

18) Y. Takagi and S. Okada, Jpn. J. Appl. Phys. 51, 085102 (2012)

19) K. Kishimoto and S. Okada, Appl. Phys. Lett. 110, 011601 (2017).

20) E. McCann, Phys. Rev. B 74, 161403 (2006).

21) E. V. Castro, K. S. Novoselov, S. V. Morozov, N. M. R. Peres, J. M. B. Lopes dos Santos, J. Nilsson, F. Guinea, A. K. Geim, and A. H. Castro Neto, Phys. Rev. 
Lett. 99, 216802 (2007).

22) M. Otani and S. Okada, J. Phys. Soc. Jpn. 79, 073701 (2010).

23) S. Konabe and S. Okada, J. Phys. Soc. Jpn. 81, 113702 (2012).

24) M. Matsubara and S. Okada, Jpn. J. Appl. Phys. 56, 075101 (2017).

25) M. Matsubara and S. Okada, Appl. Phys. Express 10, 025101 (2017).

26) P. Hohenberg and W. Kohn, Phys. Rev. 136, B864 (1964).

27) W. Kohn and L. J. Sham, Phys. Rev. 140, A1133 (1965).

28) Y. Morikawa, K. Iwata, and K. Terakura, Appl. Surf. Sci. 11, 169 (2000).

29) J. P. Perdew and Y. Wang, Phys. Rev. B 45, 13244 (1992).

30) D. M. Ceperley and B. J. Alder, Phys. Rev. Lett. 45, 566 (1980).

31) S. Okada, S. Saito, and A. Oshiyama, Phys. Rev. Lett. 86, 3835 (2001).

32) D. Vanderbilt, Phys. Rev. B 41, 7892 (1990).

33) M. Otani and O. Sugino, Phys. Rev. B 73, 115407 (2006). 